\title{
Feasibility of right-sided total extraperitoneal procedure for inguinal hernia repair after appendectomy: a prospective cohort study
}

\author{
J. W. M. Elshof · F. Keus · J. P. J. Burgmans \\ G. J. Clevers · P. H. P. Davids · T. van Dalen
}

Received: 24 June 2008 / Accepted: 24 September 2008/Published online: 5 December 2008

(c) The Author(s) 2008. This article is published with open access at Springerlink.com

\begin{abstract}
Background Totally extraperitoneal (TEP) endoscopic hernia surgery is increasingly popular since it is associated with little postoperative pain and with early return to work. Previous appendectomy may preclude preperitoneal dissection in patients with right-sided hernias. The feasibility of TEP surgery in these patients was the subject of the present study.

Methods Between January 2005 and February 2007 all consecutive patients undergoing TEP surgery were included in a prospective cohort study. The study group consisted of patients with right-sided and bilateral hernias. Operative times, conversions, complication rates, and return to daily activities were recorded. Patients were divided into two groups according to previous appendectomy.

Results A total of 462 patients with right-sided hernias underwent TEP surgery: 421 patients without previous abdominal surgery (group 1) and 41 patients with previous appendectomy (group 2). The conversion rate was significantly higher in group 2: four patients $(10 \%)$ were converted to open Lichtenstein repair versus five $(1 \%)$ in group $1(p=0.005)$. However, we found no significant differences in complication rate, operative time, and return to daily activities.

Conclusions A right-sided (or bilateral) TEP procedure may be performed safely in patients after previous appendectomy. Despite a higher conversion rate, the vast majority of patients can be operated endoscopically.
\end{abstract}

J. W. M. Elshof $(\bowtie) \cdot$ F. Keus · J. P. J. Burgmans ·

G. J. Clevers · P. H. P. Davids - T. van Dalen

Department of Surgery, Diakonessenhuis, Bosboomstraat 1,

3582, KE, Utrecht, The Netherlands

e-mail: jelshof@diakhuis.nl; jwmelshof@hotmail.com
Keywords TEP $\cdot$ Previous surgery $\cdot$ Appendectomy

The main advantages of endoscopic inguinal hernia repair are less postoperative pain and quicker convalescence when compared with conventional open hernia repair. Both transabdominal preperitoneal (TAPP) and totally extraperitoneal (TEP) hernia repair are technically demanding operative procedures necessitating adequate "surgical volume" to ensure recurrence rates comparable to the Lichtenstein procedure (Fig. 1) [1-4].

Apart form surgical expertise, previous lower abdominal surgery may be a contraindication for laparoscopic approach. Re-entering the preperitoneal space after previous surgery may preclude dissection during a total extraperitoneal hernia repair. Very few studies addressed the feasibility of TEP hernia repair following previous abdominal surgery, usually evaluating a variety of operative procedures in the lower abdomen $[5,6]$.

In a prospective cohort study we evaluated the feasibility of a right-sided TEP procedure in patients who previously underwent open appendectomy.

\section{Materials and methods}

Objective

The objective of our study was to evaluate the safety and feasibility of a right-sided or a bilateral TEP technique for inguinal hernia repair in patients following previous appendectomy. In a prospective cohort study patients who underwent a right-sided or bilateral TEP hernia repair after previous appendectomy were compared with a control group of patients with right-sided or bilateral hernias 
Fig. 1 Flow chart of patient inclusion

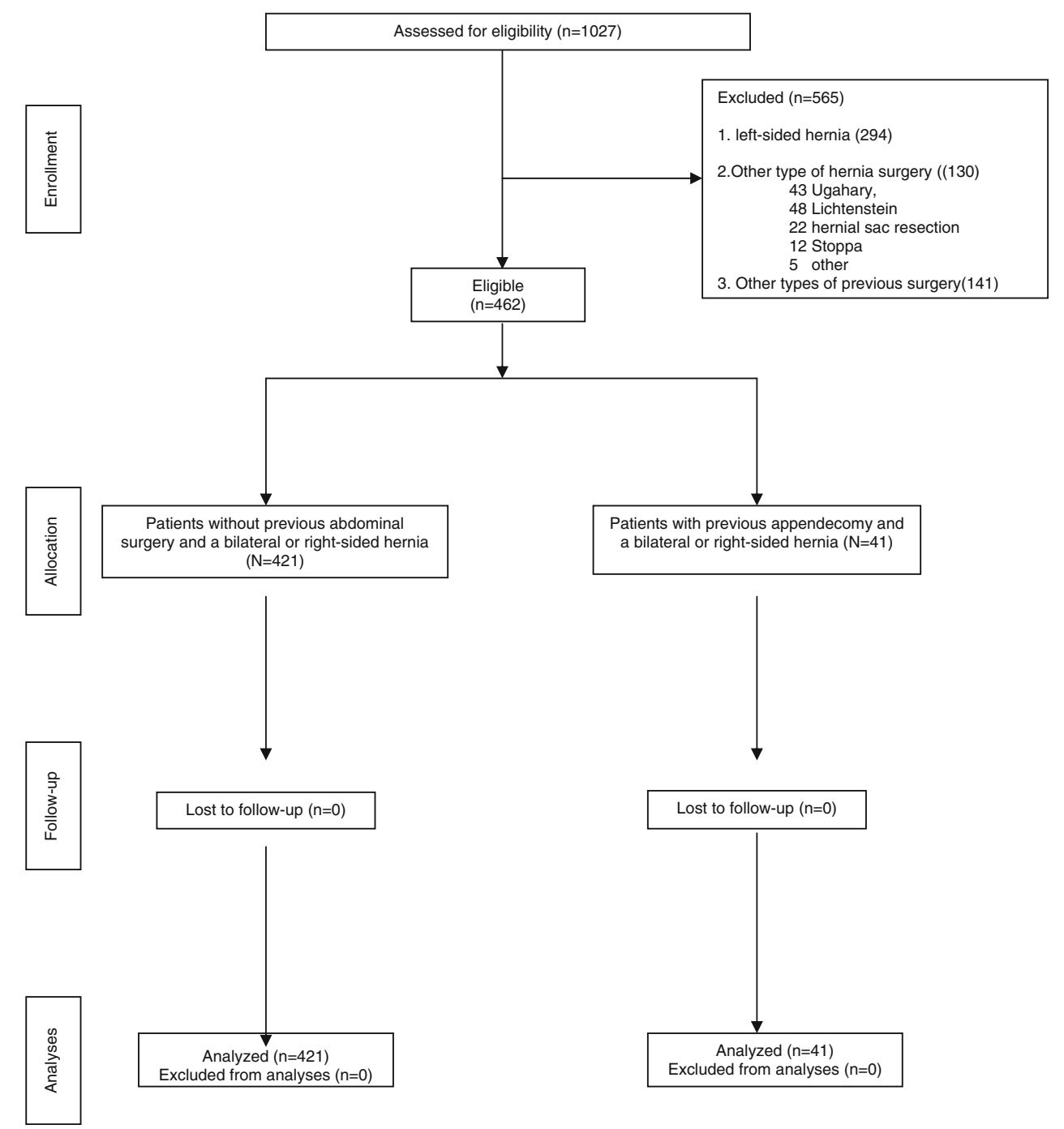

without previous abdominal surgery. We hypothesize that the TEP technique can safely be applied to patients with previous appendectomy.

Inclusion and exclusion criteria

Between January 2005 and February 2007 1,027 patients with inguinal hernia were selected for operative treatment in the department of surgery of the Diakonessenhuis. All patients diagnosed with a right-sided or bilateral inguinal hernia scheduled for TEP hernia repair were included in the study. Patients with recurrent or scrotal hernias were included in both groups. Patients with left-sided inguinal hernias and patients with an incarcerated hernia were excluded. Patients with previous appendectomy by lower midline laparotomy or laparoscopy as well as any other previous lower abdominal surgery were excluded as well.

In the Diakonessenhuis hospital three surgeons have extensive experience in the TEP technique, all three having performed more than 250 TEP procedures before 2005 . From 2005 on, TEP hernia repair was the first-choice procedure for elective hernia repair and the vast majority of patients with hernias are operated on by these three surgeons. Apart form these three surgeons, TEP surgery is not performed by any of the other seven surgeons of the surgical department.

Patients who underwent open hernia repair because they were deemed unfit for general anesthesia (a prerequisite for the TEP technique) were excluded from the study as well as patients who had an open hernia repair by one of the other staff surgeons.

\section{Surgical procedure}

Three consultant surgeons performed all operations either as the operating surgeon or supervising a surgical trainee. General anesthesia was applied in all patients. The patient was positioned on the operation table in a neutral supine 
position. The TEP technique has been described previously $[7,8]$. The approach in patients who had previously had an open appendectomy was not different from the approach in patients without previous appendectomy.

\section{Outcome measures}

Age, sex, and body mass index (BMI) were recorded prospectively. Similarly operative time, conversion to open Lichtenstein hernia repair, and intraoperative complications were registered. Postoperatively hospital stay, postoperative complications, time to return to daily activities, pain at 6 weeks postoperative, and short-term recurrences were registered by filling out forms by the patients at the 6 weeks follow-up visit.

According to previous appendectomy patients were divided into two groups: patients without previous appendectomy (group 1) and patients who had had an appendectomy through a McBurney incision (group 2).

\section{Statistical analysis}

Calculations were made using SPSS ${ }^{\circledR}$ version 13.0. For all continuous data, results are presented as both medians with ranges and as means with standard deviations. Normality of data was checked by using the Kolmogorov-Smirnov test. Levene's test was used for checking equality of variances. When the condition of normality and equal variances was met, the $t$-test was used for independent data. When equality of variances was absent or normality could not be reached, the nonparametric Mann-Whitney $U$ test was used. The chi-square test was used for dichotomous outcomes. Significance was set at a level of 0.05 .

\section{Results}

In the study period 462 patients were scheduled for TEP hernia repair for right-sided or bilateral hernia. There were 421 patients in group 1 (no previous appendectomy) and 41 patients in group 2 (previous appendectomy).

\section{Patient characteristics}

There were no significant differences in age, gender, body mass index, and the proportion of bilateral hernias between the groups (Table 1). The proportion of patients operated by a surgical trainee was not significantly different: 162 (38\%) in group 1 versus $20(49 \%)$ in group 2.

Operative results

There was no significant difference in operative time between the two groups (Table 2). The conversion rates were significantly different between both groups with five conversions to an open anterior Lichtenstein repair in group $1(1.1 \%)$ and four in group $2(9.8 \% ; p=0.005)$. The reasons for conversion in group 1 were impaired vision due to peritoneal injury $(n=3)$ and adhesions $(n=2)$. The reasons for conversion in group 2 were impaired visibility due to adhesions $(n=2)$, peritoneal injury $(n=1)$, and bladder perforation in association with adhesions $(n=1)$.

\section{Complications}

There were no significant differences in postoperative complications between the two groups (Table 3). Return to daily activities occurred after a median of 7 days (range 141 days) in group 1 and 8 days in group 2 (range 235 days). Pain sensation 6 weeks after surgery was similar

Table 1 Patient characteristics

\begin{tabular}{llll}
\hline & $\begin{array}{l}\text { Group 1: patients without } \\
\text { previous abdominal surgery }\end{array}$ & $\begin{array}{l}\text { Group 2: patients with } \\
\text { previous appendectomy }\end{array}$ & $\begin{array}{l}\text { Statistical } \\
\text { analysis }\end{array}$ \\
\hline $\begin{array}{l}\text { Total patients } \\
\text { Age (years) }\end{array}$ & 421 & 41 & \\
$\quad \begin{array}{l}\text { Mean (SD) } \\
\text { Median (range) }\end{array}$ & $54(13.8)$ & $56(12.7)$ & $p=0.572^{*}$ \\
Number of men & $55(18-87)$ & $58(30-81)$ & $p=0.384^{* *}$ \\
Body mass index (BMI)) $\mathrm{kg} / \mathrm{m}^{2}$ & $38(92)$ & $p=0.184^{* * *}$ \\
$\quad$ Mean (SD) & $24.7(2.9)$ & $24.5(3.1)$ & $p=0.392^{*}$ \\
$\quad$ Median (range) & $24.5(15.2-45.7)$ & $24.2(17.4-31.2)$ & $p=0.697^{* *}$ \\
Location of hernia & & & \\
$\quad \begin{array}{l}\text { Bilateral } \\
\text { Right sided }\end{array}$ & $164(39)$ & $19(46)$ & \\
$\quad 257(61)$ & $22(54)$ & \\
\hline Recurrent & $14(3.3)$ & $3(7.3)$ & \\
\hline
\end{tabular}


Table 2 Characteristics of operations and postoperative convalescence
Values in parentheses are percentages unless indicated otherwise

* Mann-Whitney test

** Chi-square test

Table 3 Intraoperative and postoperative complications

Values in parentheses are percentages

* Chi-square test

in both groups; there were no inguinal hernia recurrences (technical failures) in either group.

\section{Discussion}

The aim of our study was to evaluate the feasibility of the TEP procedure for right-sided inguinal hernia repair in patients with previous appendectomy. We did find a significant risk of conversion to an anterior open hernia repair in the postappendectomy group, but the chance of successful TEP hernia repair was still $90 \%$ following previous appendectomy. We did not observe an increased complication in either group, and no differences in outcome at 6 weeks follow-up.

The present study has a number of weaknesses. There is a severe imbalance between the numbers of patients in both groups. We are aware that sufficient power to detect small differences in severe adverse events (e.g., severe complications such as bladder laceration or short-term recurrences) is lacking. However, the condition of having a right-sided inguinal hernia after previous appendectomy is not very frequent, precluding any attempt to conduct the "ideal" clinical trial.
Group 1: patients without Group 2: patients with Statistical previous abdominal surgery previous appendectomy analysis $n=421 \quad n=41$
$36(14)$

$p=0.29 *$

$30(13-150)$

$30(11-70)$

$21(51)$

$p=0.19 * *$

$162(38)$

$20(49)$

$4(9.8)$

$p=0.005^{* *}$

$5(1.1)$

9 (7.46)

$11(7.60)$

$8(2-35)$

$p=0.09 *$

\begin{tabular}{lccc}
\hline Intraoperative adverse events (total) & $108(25.6)$ & $17(41.5)$ & \\
Bleeding & $16(3.7)$ & $4(9.7)$ & $p=0.09^{*}$ \\
Peritoneal injury & $90(21.2)$ & $12(29.2)$ & $p=0.24^{*}$ \\
Other & $3(0.7)$ & $1(2.6)$ & $p=0.31^{*}$ \\
Postoperative pain after 6 weeks & $75(17.8)$ & $12(29.3)$ & $p=0.11^{*}$ \\
Postoperative complications (total) & $104(24.7)$ & $18(43.9)$ & $p=0.39^{*}$ \\
Infections & $4(1.0)$ & $1(2.4)$ & $p=0.15^{*}$ \\
Hematoma & $25(5.9)$ & $5(12.2)$ &
\end{tabular}

The major advantage of our study is that bias by differential treatments is absent since these patients were all uniformly treated. Furthermore, all patients were treated by experienced surgeons.

The main reason to perform laparoscopic hernia repair is limited postoperative pain and quick convalescence. Recent randomized trials, comparing Lichtenstein with TEP hernia repair, indeed indicate less postoperative pain, shorter hospital stay, and earlier return to work associated with the TEP technique [1-3]. A Cochrane review has shown that laparoscopic hernia repair is associated with quicker convalescence [9]. On the other hand serious adverse events and early recurrences (i.e., technical failures) may be more common following laparoscopic repair $[9,10]$. In that respect the extensive learning curve of laparoscopic hernia repair is considered explanatory for the higher rate of serious adverse events following TEP hernia repair, and surgical expertise a prerequisite for obtaining good results $[10,11]$. To overcome the issue of surgical expertise we limited the performance of the operation on approximately 800 hernia patients to three surgeons with extensive laparoscopic experience.

In a department with high surgical volume we studied the feasibility of doing extraperitoneal hernia correction 
following previous dissection in the preperitoneal plane through a McBurney incision. Only two studies report TEP procedures in patients with previous lower abdominal surgery. Although both studies conclude that TEP can safely be performed in patients with a history of lower abdominal surgery, both studies report on a variety of previous abdominal surgeries and did not distinct between left- and right-sided hernia repairs $[5,6]$.

Although the rate of conversion increases considerably following previous appendectomy, still $90 \%$ may benefit from a procedure associated with considerably less pain and quicker convalescence than a Lichtenstein repair. Since the risk of serious adverse events is not increased, we feel that TEP hernia repair is the preferred operative procedure in patients with right-sided hernias and previous open appendectomy.

Acknowledgements The authors would like to thank S Elias for facilitating statistical analysis.

Open Access This article is distributed under the terms of the Creative Commons Attribution Noncommercial License which permits any noncommercial use, distribution, and reproduction in any medium, provided the original author(s) and source are credited.

\section{References}

1. Eklund A, Rudberg C, Smedberg S, Enander LK, Leijonmarck CE, Osterberg J, Montgomery A (2006) Short-term results of a randomized clinical trial comparing Lichtenstein open repair with totally extraperitoneal laparoscopic inguinal hernia repair. $\mathrm{Br} \mathrm{J}$ Surg 93:1060-1068. doi:10.1002/bjs.5405
2. Lau H, Patil NG, Yuen WK (2006) Day-case endoscopic totally extraperitoneal inguinal hernioplasty versus open Lichtenstein hernioplasty for unilateral primary inguinal hernia in males: a randomized trial. Surg Endosc 20:76-81. doi:10.1007/s00464005-0203-9

3. Lal P, Kajla RK, Chander J, Saha R, Ramteke VK (2003) Randomized controlled study of laparoscopic total extraperitoneal versus open Lichtenstein inguinal hernia repair. Surg Endosc 17:850-856. doi:10.1007/s00464-002-8575-6

4. Wake BL, McCormack K, Fraser C, Vale L, Perez J, Grant AM (2005) Transabdominal pre-peritoneal (TAPP) vs totally extraperitoneal (TEP) laparoscopic techniques for inguinal hernia repair. Cochrane Database Syst Rev 1:CD004703. doi:10.1002/ 14651858.CD004703.pub2

5. Dulucq JL, Wintringer P, Mahajna A (2006) Totally extraperitoneal (TEP) hernia repair after radical prostatectomy or previous lower abdominal surgery: is it safe? A prospective study. Surg Endosc 20:473-476. doi:10.1007/s00464-006-3027-3

6. Paterson HM, Casey JJ, Nixon SJ (2005) Totally extraperitoneal laparoscopic hernia repair in patients with previous lower abdominal surgery. Hernia 9:228-230. doi:10.1007/s10029005-0323-0

7. Arregui ME, Nagan RF (eds) (1994) Inguinal hernia, advances and controversies. Radcliffe Medical, Oxford

8. Fischer JE, Bland KI, Callery MP, Clagett GP, Jones DB, Logerfo FW, Seeger JM (eds) (2007) Mastery of surgery. Lippincott Williams \& Wilkins, Philadelphia

9. McCormack K, Scott NW, Go PM, Ross S, Grant AM, EU Hernia Trialists Collaboration (2003) Laparoscopic techniques versus open techniques for inguinal hernia repair. Cochrane Database Syst Rev 1:CD001785

10. Lamb AD, Robson AJ, Nixon SJ (2006) Recurrence after totally extraperitoneal laparoscopic repair: implications for operative technique and surgical training. Surgeon 4:299-307

11. Liem MS, van Steensel CJ, Boelhouwer RU, Weidema WF, Clevers GJ, Meijer WS, Vente JP, de Vries LS, van Vroonhoven TJ (1996) The learning curve for totally extraperitoneal laparoscopic inguinal hernia repair. Am J Surg 171:281-285. doi: 10.1016/S0002-9610(97)89569-4 